\title{
Dynamic Simulation Analysis and Optimization of Firing Rate of Rocket Launchers on Wheeled Vehicles
}

\author{
B. Van $\mathrm{Vo}^{1 *}, \mathrm{M} . \mathrm{Macko}^{2}$, D. Thai Nguyen ${ }^{1}$, \\ P. Duy Nguyen ${ }^{1}, \mathrm{H}$. Thanh Nguyen ${ }^{1}$ and T. Trong Bui ${ }^{1}$ \\ ${ }^{1}$ Department of Weapons, Le Quy Don Technical University, Hanoi, Vietnam \\ ${ }^{2}$ Department of Weapons and Ammunition, University of Defence, Brno, Czech Republic
}

The manuscript was received on 23 November 2020 and was accepted after revision for publication as research paper on 9 June 2021.

\begin{abstract}
:
The paper presents a method of determining the dynamic parameters of multiple-rocketlauncher system mounted on the wheeled vehicle based on Newton's law of motion. The dynamic simulation was conducted and then the results were compared with the corresponding experimental data to verify the reliability of the model. The model was applied in calculation and tested out on multiple-rocket-launcher system BM-21 (of Russia). The theoretical model calculation results are relatively consistent with the measured experimental data. The dependence of the launcher oscillation on the rate of fire was investigated, which determined the optimal rate of fire for each launcher. These results are used to evaluate the firing stability of the launcher when firing individual shots and firing bursts. This is an important theoretical basis which can be a reference for designers in the design process of improvement, manufacture, exploitation and use of a launcher mounted on the wheeled vehicle.
\end{abstract}

\section{Keywords:}

multiple-rocket-launcher system BM-21, multibody system dynamics, optimal rate of fire, stability of firing, wheeled vehicles

\section{Introduction}

Since the 50's of the last century, multiple-rocket-launcher systems have been researched by many scientists from developed countries in the world. Advances in materials technology, control and automation have been used to innovate, upgrade, and manufacture new generations of multiple rocket launchers to improve combat power, manoeuvrability and firing accuracy. Up to now, many generations of multiple rocket launchers have been created and they are more and more complete, promoting their

\footnotetext{
* Corresponding author: Department of Weapons, Le Quy Don Technical University, Hoang Quoc Viet 236, Hanoi, Vietnam. Phone: +420 7774015 84, E-mail:vovanbien.mta@ gmail.com
} 
power and superiority. Reality of recent border conflicts between Israel and Lebanon, Russia and Georgia, etc. has proven it.

Along with the change of fighting form, the role of the multiple rocket launcher system is increasingly enhanced in the artillery fire structure of countries. That has motivated the research, as well as the new design of the modern multiple rocket launcher systems, meeting the increasingly high requirements of combat and technology in military combat.

From the older versions, Russia and Western countries have increasingly attached great importance to the application of modern scientific and technical advances to the research, improvement, and manufacture of a series of launchers mounted on the wheeled vehicle with many types, different calibres, thereby confirming that the launcher system mounted on wheeled vehicles is a universal and preeminent equipment to meet the operational requirements in modern warfare.

For a complete and reliable theoretical basis to evaluate the quality of the launcher mounted on the wheeled vehicles after repairs, improvements, upgrades and new manufacturing, it is necessary to examine the basic factors affecting the launcher system when firing individual shots and when in burst mode. For that reason, a large number of documents, researches, modelling and simulation tools has been used as initial studies by previous researchers to investigate the underlying factors affecting the firing stability of launchers mounted on the wheeled vehicles. Some of the most noticeable documents are [1-4]. However, up to now the issue of assessing the firing stability of the multiple rocket launcher mounted on a wheeled vehicle when firing has not been studied rigorously and completely. Especially the influence of the vehicle and the road surface, the force acting on the tires and the moment characteristics have not been carefully considered. Researchers focused previously only on the stability of the launcher in the firing plane, while the factors affecting the launcher in lateral direction during firing has been neglected. Therefore, a detailed analysis on the firing stability of the launcher mounted on a wheeled vehicle during firing was thoroughly considered in this study. The multiple rocket launcher system BM-21 (of Russia) was chosen for this study, see Fig.1. The result can then be applied to all types of artillery mounted on wheeled vehicle.

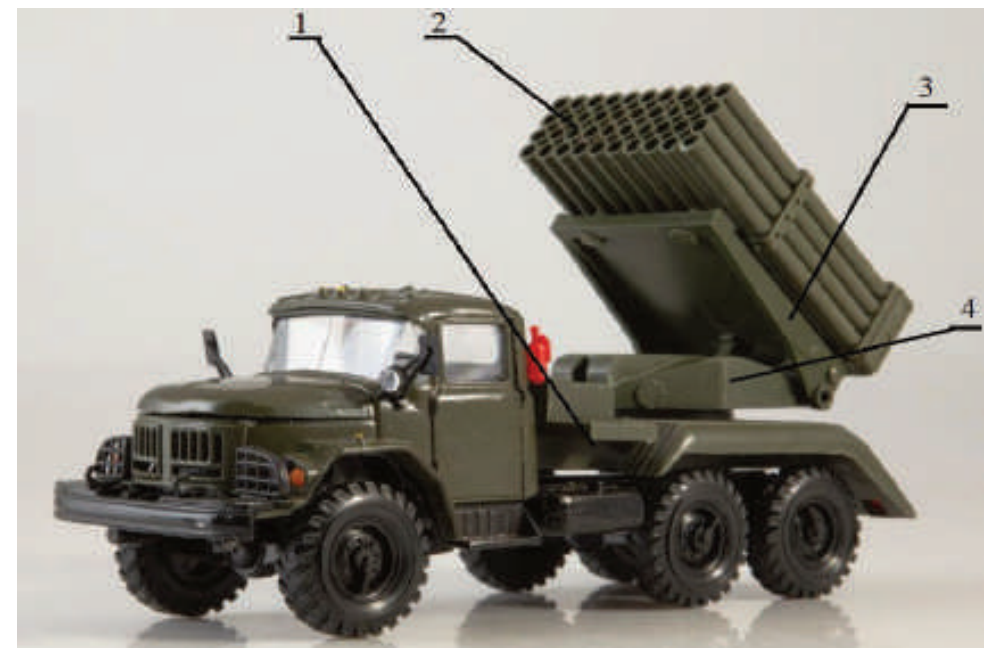

Fig. 1 Multiple-rocket-launcher system BM-21

1. Vehicle body Ural-375, 2. Launcher, 3. Elevation parts, 4. Traverser parts 
A mathematical model with 8 degrees of freedom (DOF) was used to investigate the firing stability of the multiple-rocket-launcher system in space using the Maple software. To determine the effect of the tires on the vehicle, the researchers used the Pacejka Magic Formula Tire model in [5] and [6]. This is one of the most important and widely used tire handling models. The use of this model will determine the lateral and longitudinal forces, as well as the self-aligning moment of the vehicle's tires when firing.

\section{Proposed Dynamic Model}

The dynamic model of multiple-rocket-launcher system on a combat vehicle was developed in this study. The created model includes 8 DOF using a $6 \times 6$ configuration and the model is based on Newton's law of motion. The model was developed using the integration of a few subsystems model to describe the vehicle's oscillation when firing, including: a model of vehicle oscillation in vertical plane with 5 DOF; the Pacejka's tires model; an oscillation model in the horizontal plane with 3 DOF; a lateral and longitudinal slip model and force of shot model.

\subsection{Model of Vehicle Oscillation in Vertical Plane with 5 DOF}

The oscillation model of the vehicle in vertical plane has been developed based on vertical elements of the forces acting on the mechanical system, shown in Fig. 2. In this model, the coordinate system $O X Y Z$ has the origin in the centre of sprung mass, assuming that the launcher is fixed to the vehicle floor to form vehicle body with the mass $m_{b}$, the moment of inertia around the axis $O X$ is $J_{x}$, the moment of inertia around the axis $O Y$ is $J_{y}$. When firing, the rear axle of the vehicle is locked to the vehicle's body. The front axle has the mass $m_{1}$, the moment of inertia around the axis $O_{1} X_{1}$ is $J_{x 1}$. The ground is considered absolutely rigid, suspension system and tires are represented as linear effect in the vertical direction. When firing, the force of the shot $F_{t}$ acts on the vehicle body in the direction along the launching tube axis with the traverser angle $\beta$ and the elevation angle $\varphi$. The suspension system at front right and front left has been modelled as a viscos-elastic system with stiffness and viscous resistance coefficients $k_{1}, c_{1} ; k_{2}, c_{2}$, respectively. The tires at front right and front left has a corresponding stiffness and viscous resistance coefficients $k_{01}, c_{01} ; k_{02}, c_{02}$ and the tires at rear right and rear left have a corresponding stiffness and viscous resistance coefficients $k_{03}, c_{03} ; k_{04}, c_{04}$.

The dynamic state of the mechanical system is determined with 5 DOF, including: $z$ - the longitudinal displacement of vehicle body along $O Z$-axis; $\theta$ - the angular displacement of vehicle body about $O Y$-axis; $\psi$ - the angular displacement of vehicle body about $O X$-axis; $z_{1}$ - the longitudinal displacement of the whole front axle along $O_{1} Z_{1}$ axis and $\psi_{1}-$ the angular displacement of the whole front axle about $O_{1} X_{1}$-axis.

From the mechanical model shown in Fig. 2, Newton's law of motion is used to establish a system of differential equations of motion. The equation of the 5 DOF model is represented as follows.

$$
\begin{aligned}
m_{1} \ddot{z}_{1}= & k_{1}\left[z-z_{1}-a \theta-A\left(\psi-\psi_{1}\right)\right]+c_{1}\left[\dot{z}-\dot{z}_{1}-a \dot{\theta}-A\left(\dot{\psi}-\dot{\psi}_{1}\right)\right]+ \\
& +k_{2}\left[z-z_{1}-a \theta+A\left(\psi-\psi_{1}\right)\right]+c_{2}\left[\dot{z}-\dot{z}_{1}-a \dot{\theta}+A\left(\dot{\psi}-\dot{\psi}_{1}\right)\right]- \\
& -\left[k_{01}\left(z_{1}-B \psi_{1}\right)+c_{01}\left(\dot{z}_{1}-B \dot{\psi}_{1}\right)\right]-\left[k_{02}\left(z_{1}+B \psi_{1}\right)+c_{02}\left(\dot{z}_{1}+B \dot{\psi}_{1}\right)\right]-m_{1} g
\end{aligned}
$$




$$
\begin{aligned}
& J_{x 1} \ddot{\psi}_{1}=\left\{\left[k_{01}\left(z_{1}-B \psi_{1}\right)+c_{01}\left(\dot{z}_{1}-B \dot{\psi}_{1}\right)\right]-\left[k_{02}\left(z_{1}+B \psi_{1}\right)+c_{02}\left(\dot{z}_{1}+B \dot{\psi}_{1}\right)\right]\right\} \cdot B+ \\
& +\left\{\begin{array}{l}
k_{2}\left[z-z_{1}-a \theta+A\left(\psi-\psi_{1}\right)\right]+c_{2}\left[\dot{z}-\dot{z}_{1}-a \dot{\theta}+A\left(\dot{\psi}-\dot{\psi}_{1}\right)\right]- \\
-k_{1}\left[z-z_{1}-a \theta-A\left(\psi-\psi_{1}\right)\right]+c_{1}\left[\dot{z}-\dot{z}_{1}-a \dot{\theta}-A\left(\dot{\psi}-\dot{\psi}_{1}\right)\right]
\end{array}\right\} \cdot A \\
& m_{b} \ddot{z}=-\left\{k_{1}\left[z-z_{1}-a \theta-A\left(\psi-\psi_{1}\right)\right]+c_{1}\left[\dot{z}-\dot{z}_{1}-a \dot{\theta}-A\left(\dot{\psi}-\dot{\psi}_{1}\right)\right]\right\}- \\
& -\left\{k_{2}\left[z-z_{1}-a \theta+A\left(\psi-\psi_{1}\right)\right]+c_{2}\left[\dot{z}-\dot{z}_{1}-a \dot{\theta}+A\left(\dot{\psi}-\dot{\psi}_{1}\right)\right]\right\}- \\
& -\left[2 k_{03}(z+b \theta-B \psi)+2 c_{03}(\dot{z}+b \dot{\theta}-B \dot{\psi})\right]- \\
& -\left[2 k_{04}(z+b \theta+B \psi)+2 c_{04}(\dot{z}+b \dot{\theta}+B \dot{\psi})\right]-m_{\mathrm{b}} g-F_{\mathrm{t}} \sin \varphi \\
& J_{y} \ddot{\theta}=\left\{\begin{array}{l}
\left\{k_{1}\left[z-z_{1}-a \theta-A\left(\psi-\psi_{1}\right)\right]+c_{1}\left[\dot{z}-\dot{z}_{1}-a \dot{\theta}-A\left(\dot{\psi}-\dot{\psi}_{1}\right)\right]\right\}+ \\
+\left\{k_{2}\left[z-z_{1}-a \theta+A\left(\psi-\psi_{1}\right)\right]+c_{2}\left[\dot{z}-\dot{z}_{1}-a \dot{\theta}+A\left(\dot{\psi}-\dot{\psi}_{1}\right)\right]\right\}
\end{array}\right\} \cdot a- \\
& -\left\{\begin{array}{l}
{\left[2 k_{03}(z+b \theta-B \psi)+2 c_{03}(\dot{z}+b \dot{\theta}-B \dot{\psi})\right]+} \\
+\left[2 k_{04}(z+b \theta+B \psi)+2 c_{04}(\dot{z}+b \dot{\theta}+B \dot{\psi})\right]
\end{array}\right\} \cdot b-F_{\mathrm{t}}(d \cos \varphi \cos \beta+c \sin \varphi) \\
& J_{x} \ddot{\psi}=\left\{\begin{array}{l}
\left\{k_{1}\left[z-z_{1}-a \theta-A\left(\psi-\psi_{1}\right)\right]+c_{1}\left[\dot{z}-\dot{z}_{1}-a \dot{\theta}-A\left(\dot{\psi}-\dot{\psi}_{1}\right)\right]\right\}- \\
-\left\{k_{2}\left[z-z_{1}-a \theta+A\left(\psi-\psi_{1}\right)\right]+c_{2}\left[\dot{z}-\dot{z}_{1}-a \dot{\theta}+A\left(\dot{\psi}-\dot{\psi}_{1}\right)\right]\right\}
\end{array}\right\} \cdot A+ \\
& +\left\{\begin{array}{l}
{\left[2 k_{03}(z+b \theta-B \psi)+2 c_{03}(\dot{z}+b \dot{\theta}-B \dot{\psi})\right]-} \\
-\left[2 k_{04}(z+b \theta+B \psi)+2 c_{04}(\dot{z}+b \dot{\theta}+B \dot{\psi})\right]
\end{array}\right\} \cdot B- \\
& -F_{\mathrm{t}}(d \cos \varphi \sin \beta-e \sin \varphi)
\end{aligned}
$$

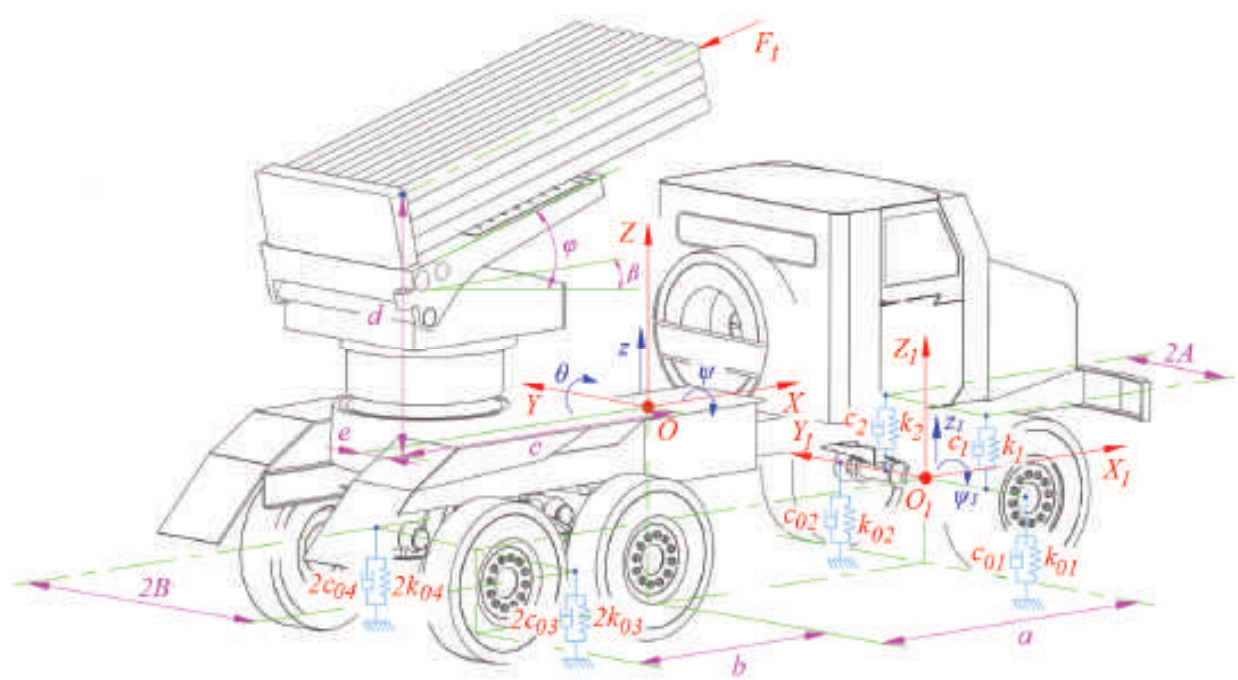

Fig. 2 Model of vehicle oscillation in vertical plane with 5 DOF 


\subsection{The Pacejka Tire Model}

The tire model is one of the main components to determine lateral and longitudinal forces, as well as self-aligning moment of tires when firing. The tire model mentioned in this study is the Pacejka Tire Model. The Magic Formula Tire model of Pacejka was introduced in 1987, developed as in [5-7], where an asphalt type of roadway was used. The model is depicted in Fig. 3.

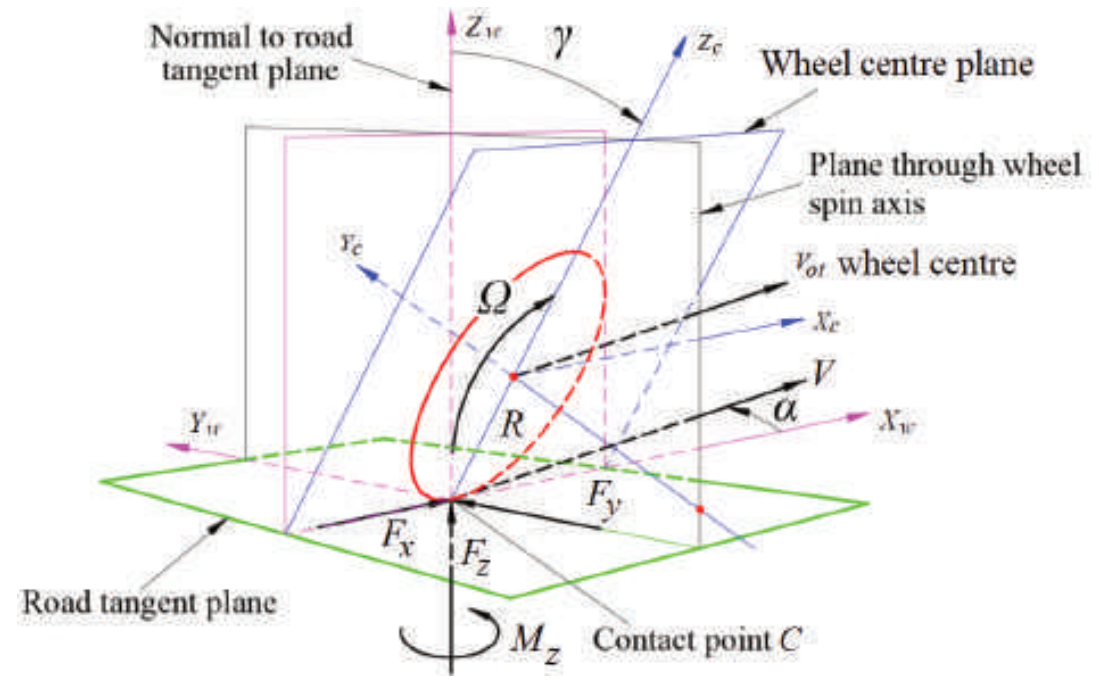

Fig. 3 Overview of the ISO axis system [7]

A general overview of the inputs and outputs of the analytical tire models is depicted in Fig. 4. The input variables of a tire model are defined on the left side, where $\lambda$ is the longitudinal slip, $\alpha$ is the lateral slip angle, $\gamma$ is the chamber angle and $F_{z}$ is the vertical load on the tire. Outputs of a tire model are the longitudinal force $F_{x}$, the lateral force $F_{y}$ and the self-aligning moment $M_{z}$, see [7].

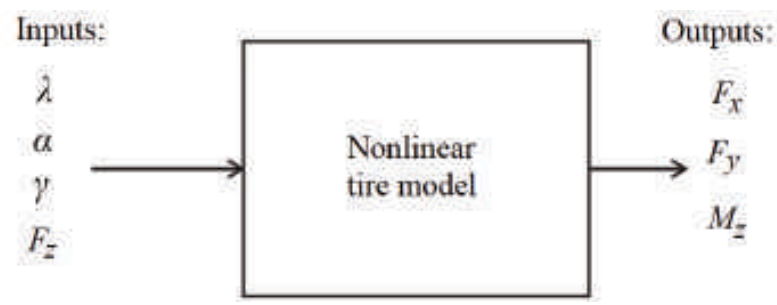

Fig. 4 Overview of the structure of the analytical tire model [7]

The general form equation of the tire forces and moment is determined by the formula, see [8]:

$$
Y(X)=y\left(x+S_{h}\right)+S_{v}
$$

where the value of $S_{h}$ and $S_{v}$ can be determined by the following formula:

$$
S_{h}=a_{9} \gamma ; \quad S_{v}=\left(a_{10} F_{z}^{2}+a_{11} F_{z}\right) \cdot \gamma
$$


The expression $y\left(x+S_{h}\right)$ representing the Pacejka Tire Model is determined by the formula:

$$
y\left(x+S_{h}\right)=D \sin \left(C \arctan \left\{B\left(x+S_{h}\right)-E\left[B\left(x+S_{h}\right)-\arctan B\left(x+S_{h}\right)\right]\right\}\right)
$$

The value $Y(X)$ is the output variable, representing the value of longitudinal force $F_{x}$, lateral force $F_{y}$ and self-aligning moment $M_{z}$. Meanwhile, the subscript $X$ is the input parameter such as lateral slip angle $\alpha$, or longitudinal slip ratio $\lambda$ of each tire of the vehicle and $\gamma$ tire chamber angle. $S_{h}$ and $S_{v}$ refer to horizontal and vertical shift of the tire response, respectively. $\alpha_{1}$ to $\alpha_{11}$ are constant parameters which are obtained from experimental analysis for type of roadway and tire, respectively [5]. The parameters $B, C$, $D$ and $E$ are defined as stiffness control, shape, peak, and curvature factor, respectively, and they can be obtained as follows, see [8]:

$$
\begin{gathered}
B=S_{t f} /(C D) \\
D=a_{1} F_{z}^{2}+a_{2} F_{z} \\
E=a_{6} F_{z}^{2}+a_{7} F_{z}+a_{8}
\end{gathered}
$$

where $S_{t f}$ is defined as the stiffness at zero slip, used to describe the lateral force:

$$
S_{t f}=a_{3} \sin \left[a_{4} \arctan \left(a_{5} F_{z}\right)\right]
$$

and, both longitudinal force as well as self-aligning moment as follows:

$$
S_{t f}=\left(a_{3} F_{z}^{2}+a_{4} F_{z}\right) / \mathrm{e}^{a_{5} F_{z}}
$$

The parameter $C$ is 1.30 for the lateral force, while for longitudinal force and selfaligning moment the parameters are 1.65 and 2.40. The model parameters are dependent on the normal force $F_{z}$ at the tires of the vehicle.

\subsection{The Oscillating Model 3 DOF in the Horizontal Plane}

The 3 DOF model in the horizontal plane mainly describes the vehicle's oscillation along the longitudinal $O X$-axis, the lateral $O Y$-axis, and the rotational motion (yaw) about the vertical $O Z$-axis. The displacements are defined as $a_{x}, a_{y}$ and $\ddot{r}$, respectively. Additionally, external forces such as longitudinal force, lateral force, $F_{t}$, and selfaligning moment acting on each tire as well as the force of the shot were also included in this model, as shown in Fig. 5.

The sum of the total forces acting on both longitudinal and lateral motion is mainly considered to formulate the longitudinal and lateral acceleration of the combat vehicle. By projecting all forces on the $x$-direction, we obtain:

$$
\sum F_{x}=m a_{x}=F_{x f r}+F_{x f l}+F_{x r r}+F_{x r l}-F_{t} \cos \varphi \cos \beta
$$

By projecting all forces on the $y$-direction, we obtain:

$$
\sum F_{y}=m a_{y}=F_{t} \cos \varphi \sin \beta+F_{y f r}+F_{y f l}+F_{y r r}+F_{y r l}
$$

Based on the longitudinal and lateral forces, as well as the self-aligning moment acting on all tires when firing, the yaw acceleration $\ddot{r}$ can be determined using the method of calculating the torque around the centre of gravity of the entire combat vehicle: 


$$
\begin{aligned}
\sum M_{y a w}= & I_{z} \ddot{r}=a\left(F_{y f r}+F_{y f l}\right)+B\left(F_{x f r}+F_{x r r}\right)-B\left(F_{x f l}+F_{x r l}\right)- \\
& -b\left(F_{y r r}+F_{y r l}\right)+\left(M_{z f l}+M_{z f r}+M_{z r l}+M_{z r r}\right)-c F_{t} \cos \varphi \sin \beta
\end{aligned}
$$

where $F_{x f r}, F_{x f l}, F_{x r r}, F_{x r l}, F_{y f r}, F_{y f l}, F_{y r r}, F_{y r l}, M_{z f r}, M_{z f l}, M_{z r r}$ and $M_{z r l}$ are the longitudinal force and lateral force, as well as the self-aligning moment of each tire at front right, front left, rear right, and rear left, respectively.

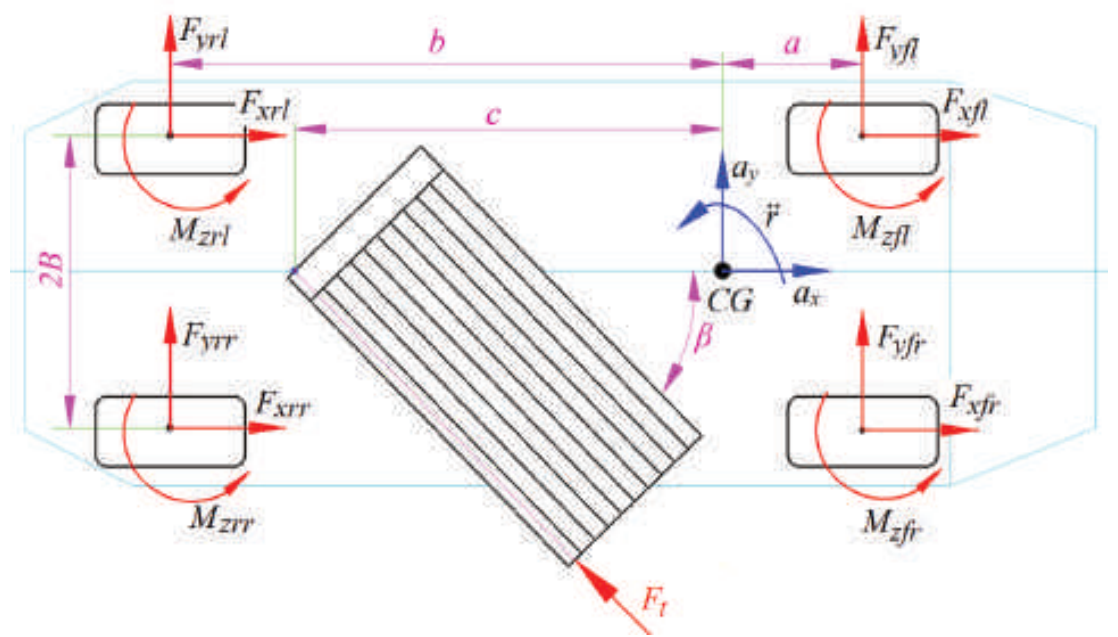

Fig. 5 Model of 3 DOF in the horizontal plane

\subsection{Lateral and Longitudinal Slip Model}

To obtain the lateral forces and longitudinal forces, as well as self-aligning moment of the tire, we need to define the input parameters in the tire model which is the lateral slip angle and longitudinal slip. The detailed explanation can be obtained from [9]. The lateral slip angle $\alpha$ is the angle between the tire direction of motion and the wheel plane, see Fig [3]. The tire lateral slip angles at the front and rear tires can be determined as follows, see [8]:

$$
\begin{aligned}
& \alpha_{f i, j}=\arctan \frac{v_{y}+a \cdot \dot{r}}{v_{x}+B \cdot \dot{r}} ; i=\text { left }, j=\text { right } \\
& \alpha_{r i, j}=\arctan \frac{v_{y}-b \cdot \dot{r}}{v_{x}+B \cdot \dot{r}} ; i=\text { left }, j=\text { right }
\end{aligned}
$$

where $\alpha_{f i, j}$ and $\alpha_{r i, j}$ are the lateral slip angles of tires at the front and rear of the vehicle. The longitudinal slip $\lambda$ of the tire is defined as the difference between the tire tangential velocity and the velocity of the axle relative to the road direction. It can be determined by the following formula:

$$
\lambda=\frac{v_{x}-R \cdot \omega}{v_{x}}
$$

where $v_{x}$ - the longitudinal velocity of the vehicle, $R$ - the radius of each wheel, $\omega-$ the angular velocity of the wheel. 


\subsection{Firing Force Model}

The firing force is the force by which the fired rocket acts on the launcher. The firing force is the main component causing vibration of the launcher. The value of the firing force is a function of time. It only depends on rocket function and does not depend on the vibrating state of the system. The firing force can be divided into three phases: the braking phase, the orientation phase, and the exhaust phase, see Fig. 6 [10].

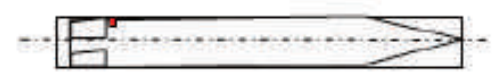

(a)

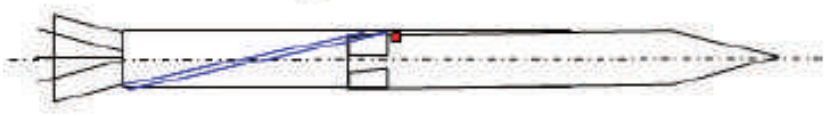

(b)

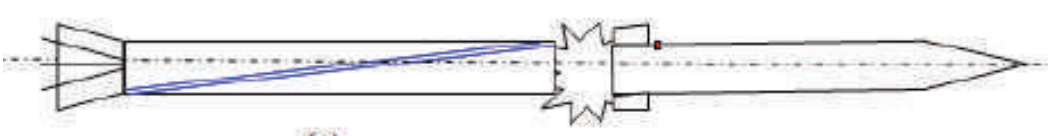

(c)

Fig. 6 Diagram of phases of firing force [10]

(a) The braking stage; (b) The orientation stage; (c) The exhaust stage

The firing force is determined as follows:

- The braking phase: when the braking force reaches the limit value $F_{k}$ (about $6 \mathrm{kN}$ to $8 \mathrm{kN}$ ), the rocket will be released. This phase is very fast in the range from 0 to $t_{k}=0.025 \mathrm{~s}$. Therefore, we assume that the braking force increases linearly from 0 to $F_{k}$ :

$$
F_{t}=F_{k} \frac{t}{t_{k}}-Q \sin \varphi ; \quad\left(0 \leq t \leq t_{k}\right)
$$

where $Q$ - is the weight of the rocket, $F_{k}$ - is the limit value of the braking force.

- The phase of rocket motion in launcher tube: This phase lasts about $0.095 \mathrm{~s}$, i.e., from $0.026 \mathrm{~s}$ until $t_{c}=0.121 \mathrm{~s}$. The actual $F_{t}$ value is not large compared to that of other phases, it is derived linearly from the value $F_{c 1}$ to $F_{c 2}$. The value of the force $F_{t}$ is determined by the formula, see [10, 11]:

$$
F_{t}=\frac{\frac{1}{m_{d}}\left(P^{\prime}-F_{k}\right)+\frac{d_{c} M_{0}}{2 I_{d x} \tan \gamma}}{\frac{d_{c}^{2}}{4 I_{d x} \tan \gamma}(\cos \gamma-f \sin \gamma)+\frac{1}{m_{d}}(\sin \gamma+f \cos \gamma)}
$$

where $P^{\prime}-$ the thrust of the engine that includes friction losses, $m_{d}$ - the mass of rocket, $M_{0}$ - the initial drag torque, $d_{c}$ - the diameter of the rocket, $I_{d x}$ - the moment of inertia of rocket around the longitudinal axis, $f$ - the friction coefficient, $\gamma$ - the slope of the rifle.

- The phase of exhaust gas acting on the launcher: when the rocket engine works, the exhaust gas flow is formed and acts on the front of the launcher. This phase 
lasts from the time the rocket comes out of the muzzle of launching tube until the exhaust gas flow stops acting on the launcher (Fig. 7). The exhaust force is determined by the following formula, see [10]:

$$
F_{t}=-\int_{(S)} \xi p(\rho, \tau) \mathrm{d} s
$$

where $S$ - the surface area of the launcher; $\mathrm{d} s$ - the differential of area; $p(\rho, \tau)-$ the gas pressure at the review point and $\xi$ - the coefficient of surface coverage.

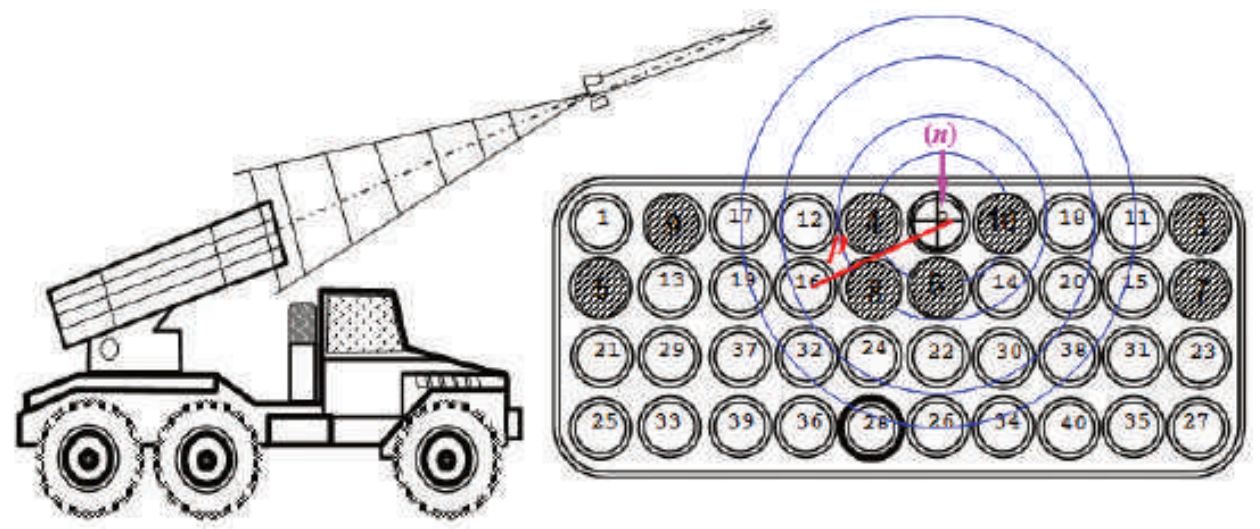

Fig. 7 Diagram of phase of gas exhaustion acting on the launcher and firing sequence

The diagram of the firing force acting on the launcher surface with respect to time is shown in Fig. 8.

\section{Validation of Model}

\subsection{Applying Model to Survey Oscillation for the Multiple Rocket Launcher System BM-21}

The mathematical model of rocket launcher on a wheeled vehicle was applied for the multiple rocket launcher system BM-21 (of Russia). The numerical values of the input parameters of the system were determined as follows: dimension parameters and mass parameters were measured directly or were obtained from technical specifications; inertia moment parameters, force point, the gravity centres coordinates were determined by Solidworks software. After each shot, mass, inertia moment and the gravity centres coordinates of vehicle body were recalculated using a subroutine. This is one of the input parameters for the next shot, see more [10]. Due to the very large numbers of inputs, only the most important are mentioned hereto, as shown in Tab 1. The main steps to solve the problem are presented in the block diagram below, see Fig. 9:

The system of differential equations was solved using the $4^{\text {th }}$ order Runge-Kutta integration method and the MAPLE programming environment. The algorithm was programmed to investigate firing options: single or series of fires. Selected results of solution are presented in Fig. 10.

The process of examining the vibration of the mechanical system shows that the semi-linkage stage directly affects the accuracy of the firing. The vibration along the $Z$-axial directly affects the elevating angle deviation, the vibration along the $Y$-axial 
directly affects the traversing angle deviation. However, the amplitude of vibration in the $Z$ direction is much larger than that in the $X$ and $Y$ directions, so the vibrating in the $Z$ direction is the main reason for the vibration of the launcher, see Fig. 10. Stemming from that reason, in this study the $Z$ direction vibration at the top of the launcher is used to compare and evaluate the rationality of the model with experimental results.

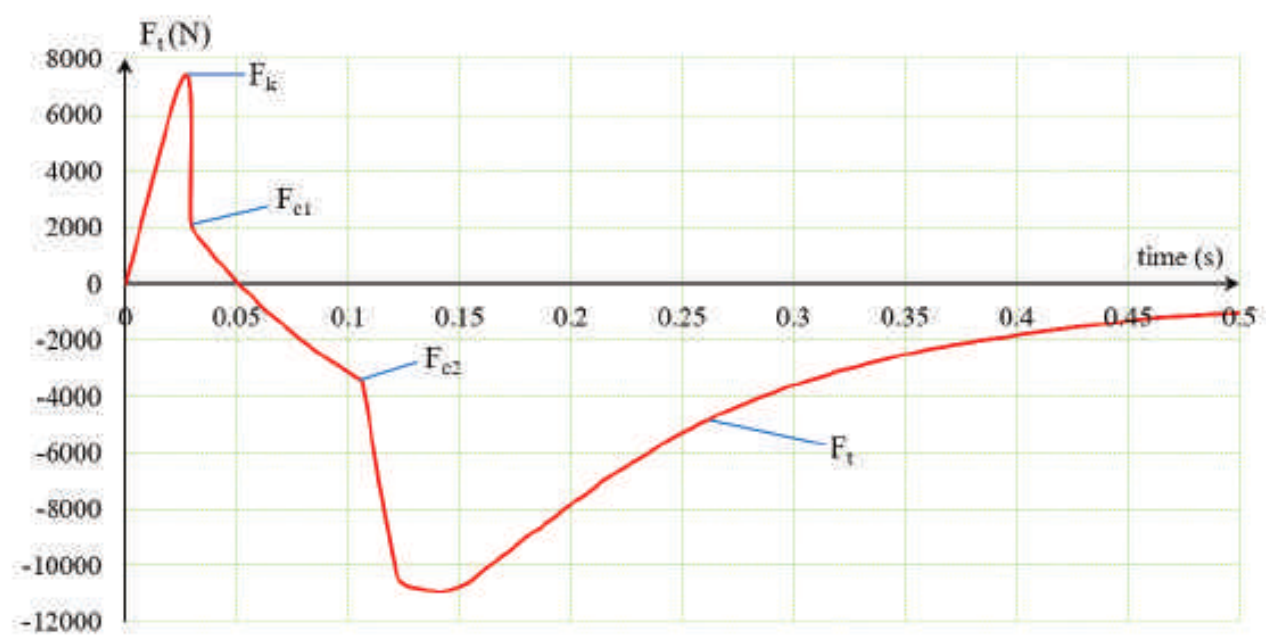

Fig. 8 Time course of firing force

Tab. 1 Parameter of multiple rocket launcher system BM-21

\begin{tabular}{|l|c|c|}
\hline \multicolumn{1}{|c|}{ Description } & Symbol & Value \\
\hline Stiffness of suspension system & $k_{1}, k_{2}$ & $165000 \mathrm{~N} / \mathrm{m}$ \\
\hline Viscous resistance coefficient of suspension system & $c_{1}, c_{2}$ & $1000 \mathrm{~N} \cdot \mathrm{s} / \mathrm{m}$ \\
\hline Tire spring stiffness & $\begin{array}{c}k_{01}, k_{02}, \\
k_{03}, k_{04}\end{array}$ & $500000 \mathrm{~N} / \mathrm{m}$ \\
\hline Viscous resistance coefficient of tire & $\begin{array}{c}c_{01}, c_{02}, \\
c_{03}, c_{04}\end{array}$ & $205000 \mathrm{~N} \cdot \mathrm{s} / \mathrm{m}$ \\
\hline Front axle mass & $m_{1}$ & $1163 \mathrm{~kg}$ \\
\hline Vehicle body mass & $m_{b}$ & $11570 \mathrm{~kg}$ \\
\hline Moment of inertia of front axle around the axis $\mathrm{X}_{1}$ & $J_{x 1}$ & $759 \mathrm{~kg} \cdot \mathrm{m}^{2}$ \\
\hline Moment of inertia of vehicle body around the axis X & $J_{x}$ & $7639.8 \mathrm{~kg} \cdot \mathrm{m}^{2}$ \\
\hline Moment of inertia of vehicle body around the axis Y & $J_{y}$ & $39497.9 \mathrm{~kg} \cdot \mathrm{m}^{2}$ \\
\hline \multirow{2}{*}{ Distances in Figs 2 and 5 } & $a$ & $3.2 \mathrm{~m}$ \\
\cline { 2 - 3 } & $b$ & $0.7 \mathrm{~m}$ \\
\cline { 2 - 3 } & $c$ & $2.18 \mathrm{~m}$ \\
\cline { 2 - 3 } & $d$ & $1.255 \mathrm{~m}$ \\
\cline { 2 - 3 } & $e$ & $0.715 \mathrm{~m}$ \\
\cline { 2 - 3 } & $A$ & $0.825 \mathrm{~m}$ \\
\cline { 2 - 3 } & $B$ & $1.15 \mathrm{~m}$ \\
\hline
\end{tabular}




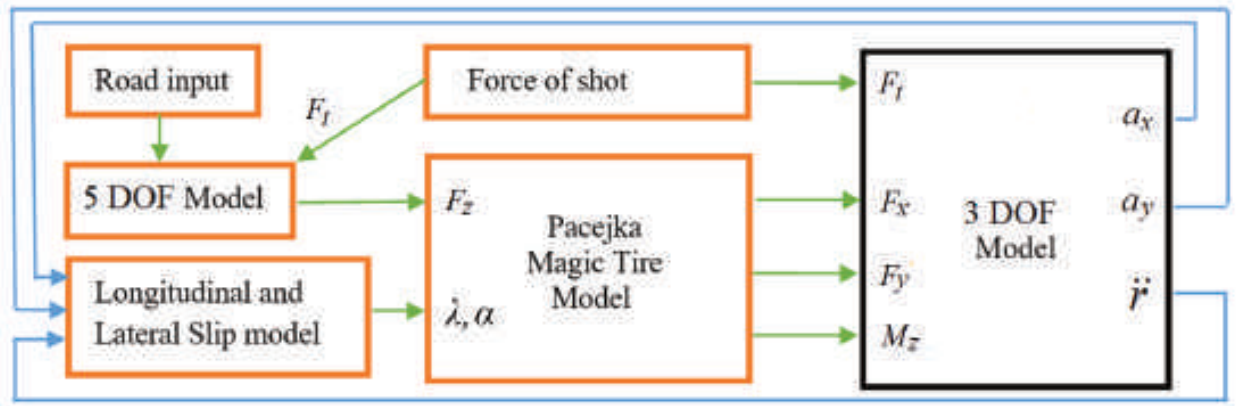

Fig. 9 General block diagram for solving the problem

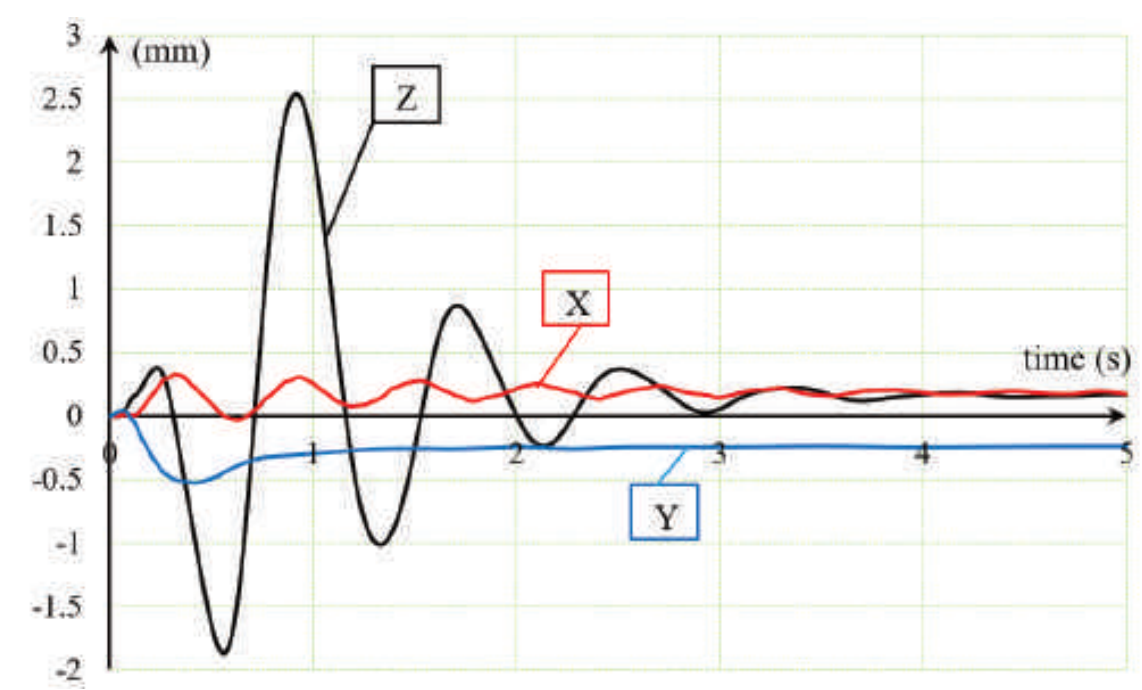

Fig. 10 Vibrating in the $X, Y, Z$ directions of the top of the launcher tube number 1 with $\alpha=25^{\circ}, \varphi=30^{\circ}, S_{d}=\{$ all 40 rockets $\}, S_{b}=\{1\}, T_{\mathrm{s}}=0.5 \mathrm{~s}$

\subsection{Experiments Determining Oscillation of the Multiple Rocket Launcher System BM-21}

The purpose of the experiment is to verify the mathematical model mentioned above. The measured oscillation parameters are the vibration of the launcher in the $X$ direction, $Y$ direction and $Z$ direction. The detailed experimental model of measuring oscillation of the multiple rocket launcher system BM-21 is presented in the document [12]. A diagram depicting the experimental equipment system is shown in Fig. 11. From the experience, the estimated maximum frequency of the mechanical system was set at $300 \mathrm{~Hz}$, and so a $20000 \mathrm{~Hz}$ sampling frequency was sufficient for the purpose of signal digitalization. Before digitalization, the signals were filtered by low-frequency filters with low-pass filter at $120 \mathrm{~Hz}$.

Some pictures from the experiment of BM-21 combat vehicle at the shooting range are shown in Figs 12 and 13. The experiment was conducted under the temperature of $25 \div 31^{\circ} \mathrm{C}$ and the humidity of $65 \%$. 


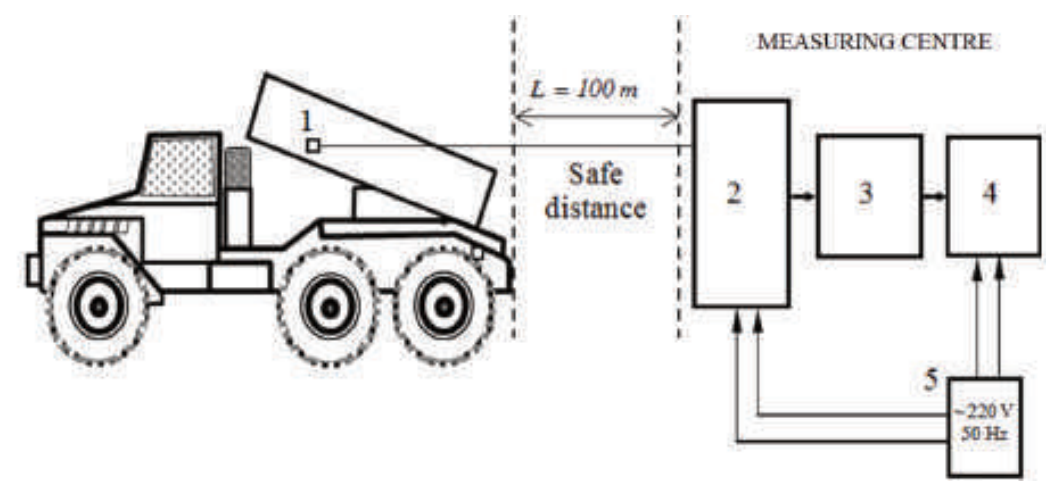

Fig. 11 The launcher and sensor gauge

1. 3D acceleration measurement sensor gauge BR4321V; 2 . Vibration measurement module UV-05A; 3. Display module UV-12A; 4. Computers and specialized software, 5. AC power source $220 \mathrm{~V}, 50 \mathrm{~Hz}$.

After the measurements have been made, the sensor gauge outputs the data file as a pair of columns of time and displacement data. Measured data are analysed and processed by DASYLab software.

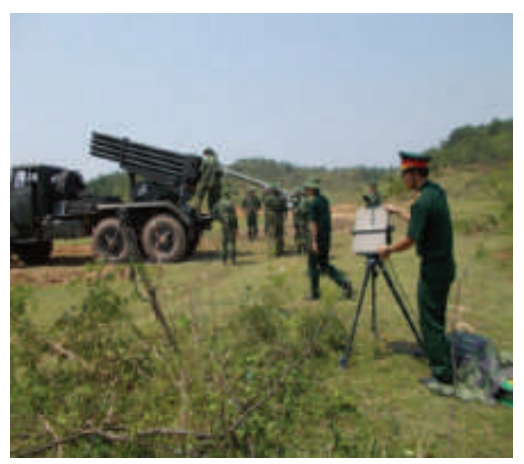

Fig. 12 Deployment of experiment

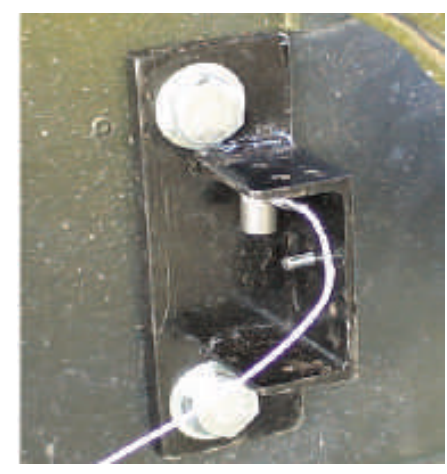

Fig. 13 Arrangement of vibration sensor

\subsection{Results and Discussion}

In order to compare the compatibility of the established model and the experimental results of the vibration measurement of BM-21 combat vehicles when firing, the firing option and experimental plan are selected as follows: a load of 10 rockets in the order $S_{d}=\{1, \ldots, 10\}$, single firing in order of 1 to $8 S_{b}=\{i, i=1, \ldots, 8\}$, firing a series of 2 rockets $S_{b}=\{i, i=9,10\}$ from the remaining rockets with the time between 2 shots equal to $5 \mathrm{~s}$. The elevation angle is $\varphi=25^{\circ}$ and traverser angle is $\beta=30^{\circ}$. The deviation between calculation results and experimental results corresponding to the single firing and a series of firing are shown in Figs 14 and 15.

The comparisons show a very good agreement between the results of calculation and experimental results. In the first cycle, the amplitude of vibration calculated according to the model and the experimental results are relatively similar. The deviations of the amplitude of vibration are within the permitted limits $(<7 \%)$ and the average deviation for the first 1000 measurement points is relatively small $(<8 \%)$. 


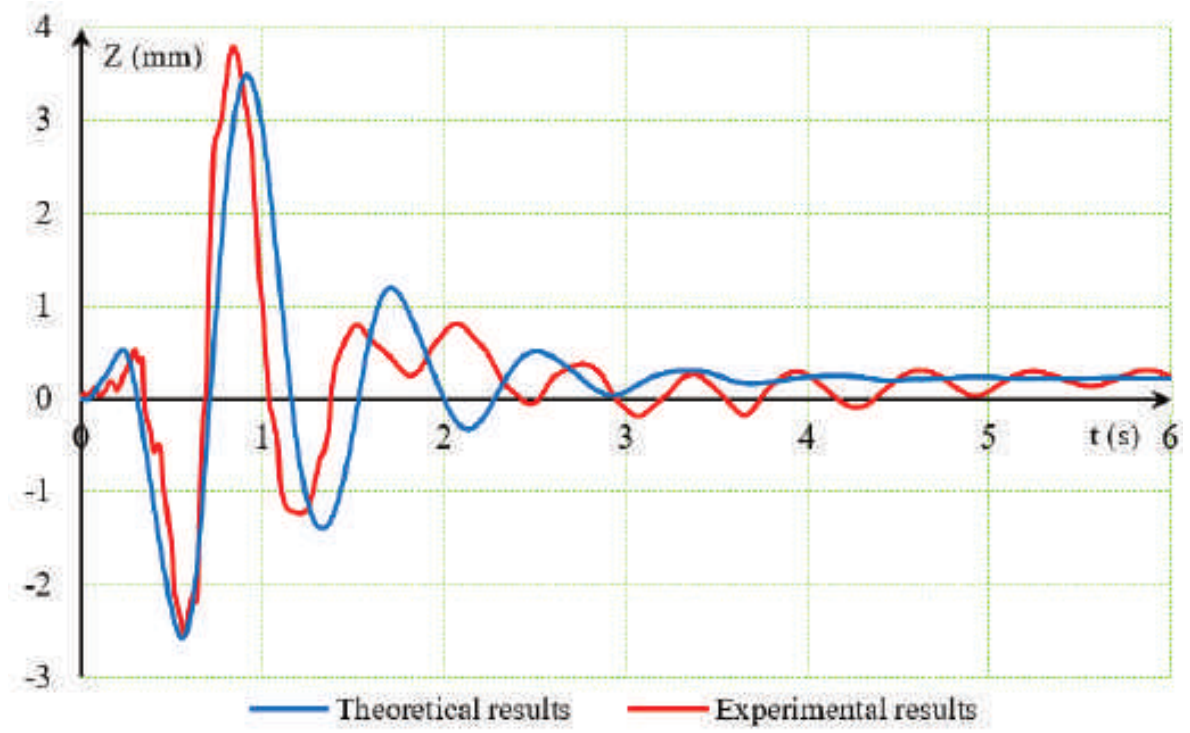

Fig. 14 The graph of vibration in the $Z_{0}$-direction when the first rocket is fired

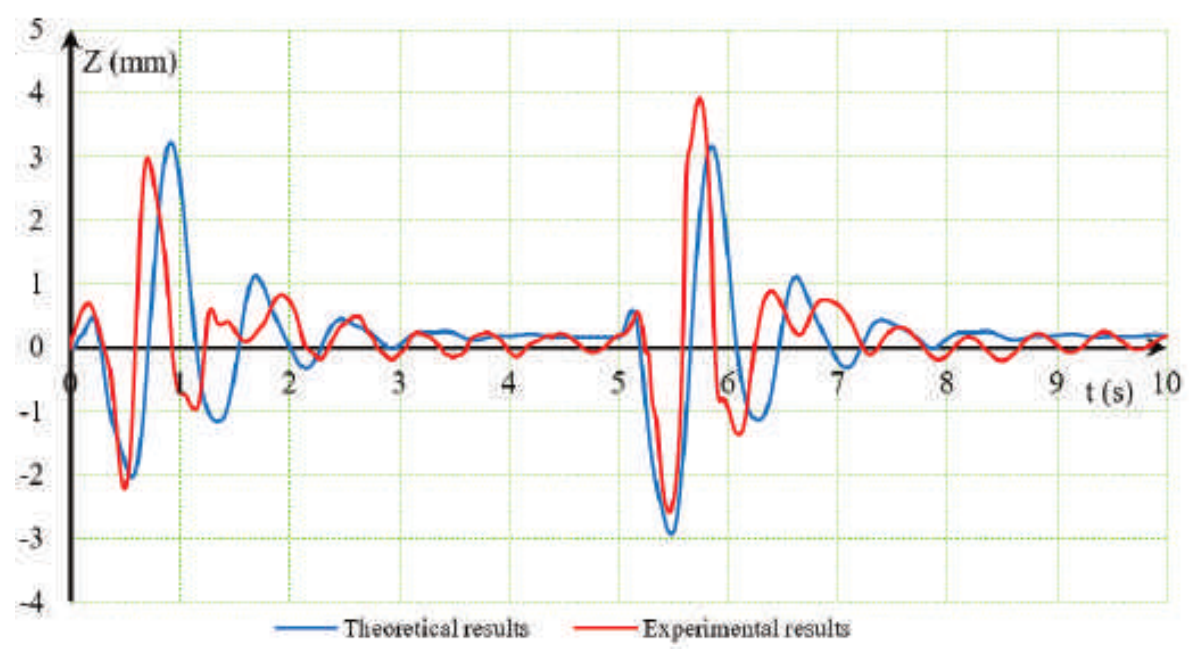

Fig. 15 The graph of vibration in the $Z_{0}$-direction when firing a series of 2 rockets number 9-10

In the period from 1 to 2 seconds, the amplitude of vibration calculated according to the model and the experimental results differ greatly. The reason for this difference is the natural disturbances acting on the launcher when fired. When the main oscillation of the launcher fades, the vibration caused by the disturbances is significant.

The analysis results show that the established model of the mechanical system mounted on the wheeled vehicles is completely consistent with reality. This model can be used for survey and evaluating the structural parameters of the launchers to improve firing accuracy, as well as to evaluate the quality of the launchers after repairs and improvements. 


\section{Optimization of Firing Rate of Rocket Launchers on a Wheeled Vehicles}

To research the time delay $T_{\mathrm{s}}$ (the time from a shot to the next) between two consequent unguided rocket launches, we are only interested in the case of series of shots. The time delay is the basis used to determine the interaction between the rockets when launched through the vibration of the launcher. In combat, to ensure firepower focus, the firing rate of the launcher needs to be increased. However, the motion of unguided rocket is also influenced by the stirred-up gas exhaust from the previous rocket. These are factors that significantly affect the unguided rocket firing accuracy. Therefore, the task of the optimal time delay determination (rate of fire) for the launcher is essential and must be considered. Obviously, the first launch has a bounce angle of the launcher unchanged with every time delay. So, to evaluate the quality of the series of shots, we use statistical methods for 4 rockets from 2-nd rocket to 5-th rocket, including sample mean $(\bar{x})$ and sample standard deviation $\left(\sigma_{x}\right)$. They are determined by the following formulas, see [13]:

$$
\begin{gathered}
\bar{x}=\frac{x_{1}+x_{2}+\ldots+x_{n}}{n}=\frac{1}{n} \sum_{i=1}^{n} x_{i} \\
\sigma_{x}=\sqrt{\frac{\sum_{i=1}^{n}\left(x_{i}-\bar{x}\right)^{2}}{n-1}}
\end{gathered}
$$

where: $x_{i}$ - the sample data set; $n$ - the sample size.

According to the article [14-16], the firing stability evaluation criterion is the jumping angle of the weapon in the vertical plane. So, in this study, we selected the bounce angle of the launcher in the vertical plane as optimal conditions to evaluate the effect of the time delay on firing stability of the multiple rocket launcher system when firing burst.

To examine the effects of the time delay $T_{\mathrm{s}}$ on the firing stability of launcher mounted on the wheeled combat vehicle, the authors only evaluated the case of firing a series of 5 rockets with time delay $T_{\mathrm{s}}$ changing from $0.4 \mathrm{~s}$ to $0.6 \mathrm{~s}$ (step equals $0.02 \mathrm{~s}$ ). The elevation angle is $\varphi=25^{\circ}$ and the traverser angle is $\beta=30^{\circ}$. The results the bounce angle values at the time the rocket leaves the launcher tube muzzle (at $t=0.121 \mathrm{~s}$ ) are presented in Fig. 16 and Tab. 2.

The obtained results can be interpreted as follows:

From Fig. 16 and Tab. 2, we can see that the value of the bounce angle is of random quantity. Minimum average deviation in time delay is $T_{\mathrm{s}}=0.44 \mathrm{~s}\left(\bar{\theta}=0.211 \times 10^{-3}\right.$ $\mathrm{rad})$, but minimum mean square deviation in time delay is $T_{\mathrm{s}}=0.5 \mathrm{~s}\left(\sigma_{\theta}=0.482 \times 10^{-3}\right.$ $\mathrm{rad})$ and $T_{\mathrm{s}}=0.6 \mathrm{~s}\left(\sigma_{\theta}=0.581 \times 10^{-3} \mathrm{rad}\right)$.

Bounce angle is stable at the time delay $T_{\mathrm{s}}=0.50 \mathrm{~s}$ and converges accordingly with the period $t=0.1 \mathrm{~s}$, which means that they will stabilize at the time delay $T_{\mathrm{s}}=0.60 \mathrm{~s}, T_{\mathrm{s}}=0.70 \mathrm{~s}$, etc. But to ensure firepower focus, we need to increase the firing rate of the launcher, so $T_{\mathrm{s}}=0.5 \mathrm{~s}$ is the best time delay. This explains why the multiple rocket launcher system BM-21 has the time delay $T_{\mathrm{s}}=0.5 \mathrm{~s}$ (rate of file $=$ 2 rounds/s), which is considered the optimal time delay.

Depending on the firing mode (reload mode, shot order, angle shot, etc.), an acceptable time delay can take $T_{\mathrm{s}}=0.5 \pm 0.01 \mathrm{~s}, 0.6 \pm 0.01 \mathrm{~s}, 0.7 \pm 0.01 \mathrm{~s}$, with the standard deviation of the angle of bounce after 4 shots not exceeding $\left[\sigma_{\theta}\right]=0.6 \times 10^{-3} \mathrm{rad}$. 


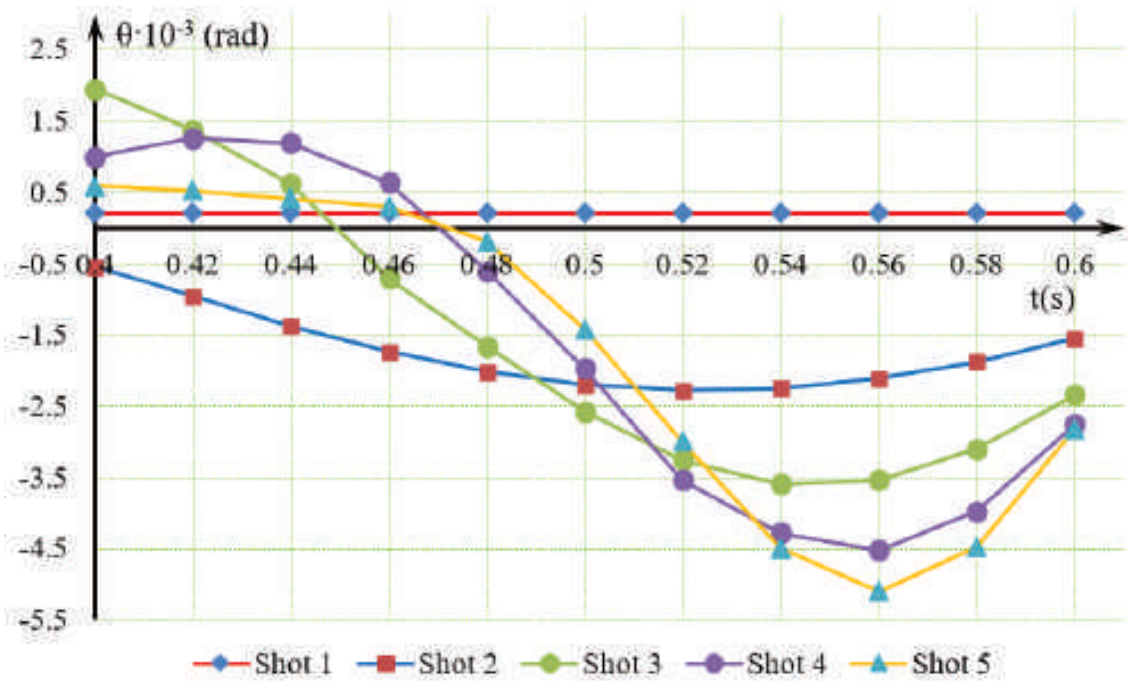

Fig. 16 The bounce angle values $\theta$ in the vertical plane corresponding to different time delays of the first 5 launches

Tab. 2 The bounce angle values $\theta \times 10^{-3}$ [rad] of the first 5 launches

\begin{tabular}{|c|c|c|c|c|c|c|c|}
\hline $\begin{array}{c}T_{s} \\
{[\mathrm{~s}]}\end{array}$ & $\begin{array}{c}\text { Shot 1 } \\
\left(\theta_{1}\right)\end{array}$ & $\begin{array}{c}\text { Shot 2 } \\
\left(\theta_{2}\right)\end{array}$ & $\begin{array}{c}\text { Shot 3 } \\
\left(\theta_{3}\right)\end{array}$ & $\begin{array}{c}\text { Shot 4 } \\
\left(\theta_{4}\right)\end{array}$ & $\begin{array}{c}\text { Shot 5 } \\
\left(\theta_{5}\right)\end{array}$ & $\left(\bar{\theta}_{(4)}\right)$ & $\left(\sigma_{\theta(4)}\right)$ \\
\hline 0.40 & 0.206 & -0.553 & 1.943 & 0.997 & 0.582 & 0.742 & 1.034 \\
\hline 0.42 & 0.206 & -0.956 & 1.367 & 1.253 & 0.519 & 0.546 & 1.069 \\
\hline 0.44 & 0.206 & -1.374 & 0.616 & 1.189 & 0.414 & 0.211 & 1.107 \\
\hline 0.46 & 0.206 & -1.736 & -0.707 & 0.637 & 0.296 & -0.378 & 1.071 \\
\hline 0.48 & 0.206 & -2.017 & -1.67 & -0.606 & -0.198 & -1.123 & 0.861 \\
\hline 0.50 & 0.206 & -2.201 & -2.574 & -1.971 & -1.426 & -2.043 & 0.482 \\
\hline 0.52 & 0.206 & -2.284 & -3.253 & -3.536 & -2.996 & -3.017 & 0.536 \\
\hline 0.54 & 0.206 & -2.249 & -3.591 & -4.285 & -4.492 & -3.654 & 1.013 \\
\hline 0.56 & 0.206 & -2.114 & -3.533 & -4.524 & -5.101 & -3.818 & 1.308 \\
\hline 0.58 & 0.206 & -1.876 & -3.096 & -3.972 & -4.473 & -3.354 & 1.138 \\
\hline 0.60 & 0.206 & -1.558 & -2.344 & -2.758 & -2.816 & -2.369 & 0.581 \\
\hline
\end{tabular}

\section{Conclusion}

The stability of the firing is one of the important problems when calculating and designing the weapons system, since it has a great influence on firing accuracy. The paper presents the method of determining some indications to evaluate the firing stability of multiple rocket launcher system mounted on the wheeled vehicle by modelling method based on Newton's law of motion and experimental methods. The model was built for multiple rocket launcher system BM-21 (of Russia) with 8 degrees of freedom for single and multiple shots. The comparison of theoretical and experimental results 
shows a very good accuracy and reasonableness of the model. The dependence of the launcher oscillation on the rate of fire was investigated, which determined the optimal rate of fire for each launcher. This model is applicable to all guns, artillery as well as launchers mounted on the wheeled vehicle. These results may be utilised in the design process to help optimize the structure of combat vehicles - combine weapons.

\section{Acknowledgment}

The work presented in this paper has been supported by the Weapon Technology Centre and Faculty of Weapons, Le Quy Don Technical University in Hanoi and by research project of the Ministry of Defence and by the Specific Research Support Project of the department weapon and ammunition K201, Faculty of Military Technology, University of Defence, Brno.

\section{References}

[1] DZIOPA, Z. and M. NYCKOWSKI. Modal Analysis of a Discrete System in the Form of a Rocket Launcher Installed on a Motor Vehicle. Problems of Mechatronics Armament Aviation Safety Engineering, 2016, 7(3), pp. 47-58. DOI 10.5604/ 01.3001.0009.2981.

[2] DZIOPA, Z., I. KRZYSZTOFIK and Z. KORUBA. An Analysis of the Dynamics of Launcher-Missile System on a Moveable Base. Bulletin of the Polish Academy of Sciences Technical Sciences, 2010, 58(4), pp. 645-650. DOI 10.2478/v10175010-0068-5.

[3] SHAPIRO, Ya.M., G.Yu. MAZING and N.E. PRUDNIK. Solid Fuel Rocket Design Basics (in Russian). Moscow: Military Publishing House, 1968.

[4] RAYZBERG, B.A., B.T. EROKHIN and K.P. SAMSONOV. Fundamentals of the Theory of Workflows in Solid Propellant Rocket Systems (in Russian). Moscow: Mechanical Engineering, 1972.

[5] WONG, J.Y. Theory of Ground Vehicles. $3^{\text {rd }}$ ed. Ottawa: Wiley, 2001. ISBN 978-0471-35461-9.

[6] PACEJKA, H. Tire and Vehicle Dynamics. $2^{\text {nd }}$ ed. Oxford: ButterworthHeinemann, 2005. ISBN 978-0-7506-6918-7.

[7] UIL, R.T. Tire Models for Steady-State Vehicle Handling Analysis [Master's Thesis]. Eindhoven: Eindhoven University of Technology, 2007.

[8] APAROW, V.R., K. HUDHA, M.M. HAMDAN and S. ABDULLAH. Study on Dynamic Performance of Armoured Vehicle in Lateral Direction due to Firing Impact. Advances in Military Technology, 2015, 10(2), pp. 5-20. ISSN 2533-4123.

[9] APAROW, V.R., F. AHMAD, K. HUDHA and H. JAMALUDDIN. Modelling and PID Control of Antilock Braking System with Wheel Slip Reduction to Improve Braking Performance. International Journal of Vehicle Safety, 2013, 6(3), pp. 265296. DOI 10.1504/IJVS.2013.055025.

[10] VO, V.B. Survey of the Dynamic Problem of the BM 21 Multiple Rocket Launcher When Taking into Account Some Random Disturbances (in Vietnamese) [Master's Thesis]. Hanoi: Military Technical Academy, 2016. 
[11] VO, V.B., S. BEER, S.T. NGO, P.D. NGUYEN. The Effect of the Nozzle Ultimate Section Diameter on Interior Ballistics of HV-76 Trial Gun. In: Proceedings of the International Conference on Military Technologies 2019. Brno: IEEE, 2019. DOI 10.1109/MILTECHS.2019.8870040.

[12] PHAM, H.S. Study the Dynamics of Multiple Rocket Launchers on the Combat Vehicle (in Vietnamese) [PhD Thesis]. Hanoi: Military Technical Academy, 2011.

[13] LEHMANN, L.E. and G. CASELLA. Theory of Point Estimation. $2^{\text {nd }}$ ed. New York: Springer, 1998. ISBN 978-0-387-98502-6.

[14] BALLA, J., M. HAVLICEK, L. JEDLICKA, Z. KRIST and F. RACEK. Firing Stability of Mounted Small Arms [online]. International Journal of Mathematical Models and Methods in Applied Sciences, 2011, 5(2), pp. 412-422. [viewed 202002-26]. Available from: https://www.naun.org/main/NAUN/ijmmas/19-818.pdf

[15] NGO, T.S., S. BEER, B.V. VO, P.D. NGUYEN and P.M. NGUYEN. Oscillation of the Anti-tank Missile System Fagot Fired on the Elastic Ground. In: Proceedings of the International Conference on Military Technologies 2019. Brno: IEEE, 2019. DOI 10.1109/MILTECHS.2019.8870069.

[16] NGUYEN, T.H. and B.V. VO. Evaluation of the Effect of the Thrust Deviation on the BM-21 Launcher's Oscillation in a Single Volley (in Vietnamese). Journal of Science and Technology, 2016, $\mathrm{N}^{0} 179$, pp. 132-139. ISSN 1859-0209. 